\title{
Averaged Control for Fractional ODEs and Fractional Diffusion Equations
}

\author{
Darko Mitrovic $\mathbb{D D}^{1}{ }^{1}$ Andrej Novak, ${ }^{2}$ and Tarik Uzunovic ${ }^{3}$ \\ ${ }^{1}$ Faculty of Mathematics, University of Montenegro, George Washington st. bb, 81000 Podgorica, Montenegro \\ ${ }^{2}$ Faculty of Science, Department of Physics, Division of Theoretical Physics, University of Zagreb, Bijenicka cesta 30, \\ 10000 Zagreb, Croatia \\ ${ }^{3}$ Faculty of Electrical Engineering, University of Sarajevo, Zmaja od Bosne bb, 71000 Sarajevo, Bosnia and Herzegovina \\ Correspondence should be addressed to Darko Mitrovic; darkom@ac.me
}

Received 27 May 2018; Accepted 11 July 2018; Published 17 July 2018

Academic Editor: Gisele Mophou

Copyright (C) 2018 Darko Mitrovic et al. This is an open access article distributed under the Creative Commons Attribution License, which permits unrestricted use, distribution, and reproduction in any medium, provided the original work is properly cited.

We generalize results concerning averaged controllability on fractional type equations: system of fractional ODEs and the fractional diffusion equation. The proofs are accomplished by introducing appropriate Banach space in which we prove observability inequalities.

\section{Introduction and Notation}

General problem of control theory amounts to choosing certain control which would lead a system (e.g., governed by a system of (partial) differential equations) from the given initial state to the prescribed final state. Such types of problems have obviously great potential in the sense of various applications and, thus, the control theory is very well developed. It is hard to say where the first mathematical treatment of the problem essentially originates but one can find lots of information in the standard books, e.g., [13].

However, in certain situation it is not possible to precisely determine the coefficients governing the process and it is natural that the coefficients depend on another (essentially stochastic) variable. In such a situation, we cannot require exact controllability of the system but so-called average controllability introduced first in [4] (to the best of our knowledge).

After that, several interesting publications appeared, e.g., [5-8] and the Ph.D. thesis [9], which will be in the basis of our approach. In the current contribution, we shall extend mentioned results concerning the averaged control (in particular, those from $[4,9])$ on equations containing fractional derivatives.
In the first part of the paper, we shall deal with the following finite dimensional system of ODEs:

$$
\begin{aligned}
& \partial_{t}^{\alpha} y(t, v)=A(\nu) y(t, \nu)+B(\nu) u(t), \\
& \alpha=1+\epsilon \in(1,2), \\
& \partial_{t}^{\epsilon} y(0, \nu)=y^{0} \in \mathbb{R}^{d},
\end{aligned}
$$

where $\partial_{t}^{\alpha}$ is the fractional derivative of order $\alpha \geq 1$ (to be defined later), $y(t, v) \in \mathbb{R}^{d}$ is the state of the system, $A(\nu) \in$ $M^{d \times d}$ and $B(\nu) \in M^{d \times N}$ are given matrices depending on the (stochastic) variable $v \in(0,1)$, and $y_{0} \in \mathbb{R}^{d}$ is the given initial state. The control problem here is to find the vector-valued function $u \in L^{2}\left(\mathbb{R}^{+} ; \mathbb{R}^{N}\right)$ such that, for the given final state $y_{1} \in \mathbb{R}^{d}$ (it is independent of $\nu \in(0,1)$ ), we have

$$
\int_{0}^{1} \partial_{t}^{\epsilon} y(T, \nu) d \nu=y_{1} \in \mathbb{R}^{d}
$$

for the final time $T$. Remark that here we have the Lebesgue integration with respect to $v \in \mathbb{R}$. However, there are no obstacles to choose integration with respect to some probability measure as we shall do in the next part (see $[6,9])$. In order to get the result, we shall adapt the method from [4]. More precisely, we shall rewrite the controllability problem 
into the observability one and then prove the observability estimate.

We remark that we could also consider the standard initial and final data (without the $\epsilon$-derivative), but it is more standard and convenient for the fractional ODEs to consider the type of initial data given in (1) (see, e.g., [10]).

In the second part of the paper, we shall consider the fractional diffusion equation which has received considerable attention recently (see, e.g., [11] and references therein). In our case, it has the form (see [9])

$$
\begin{aligned}
\partial_{t} y(t, \mathbf{x}, v) & =A(\nu) \Delta^{\alpha} y(t, \mathbf{x}, v)+B(\nu) u(t, \mathbf{x}) \\
t \in(0, T) & \\
y(0, \mathbf{x}, \nu) & =y_{0}(\mathbf{x}) \in V
\end{aligned}
$$

for an appropriately chosen Hilbert space $V$. Here, $A$ is known positive bounded function (or stochastic variable defined on a probability space $(\Omega, \mathscr{F}, P)), B(\omega): L^{2}\left(\mathbb{R}^{d}\right) \rightarrow V$ is a bounded operator for every $(t, v) \in \mathbb{R}^{+} \times \mathbb{R}$, the function $y_{0}$ is given, and $\Delta^{\alpha}$ is the fractional Laplacean (to be defined later). As before, we are looking for the control function $u \in$ $L^{2}\left((0, T] \times \mathbb{R}^{d}\right)$ such that, for the given (final) state $y_{1} \in V$, it holds that

$$
\int_{\mathbb{R}} y(T, \mathbf{x}, v) d P(\nu)=y_{1}
$$

for the final time $T>0$. Unlike the situation from [9] where the initial-boundary value problem for the diffusion equation is considered, here we deal with the Cauchy problem which, by means of the Fourier transform, provides us with observability estimates under appropriate assumptions on the relation between $A$ and $B$.

The paper is organized as follows. After the Introduction, we recall the notions and notations that we are going to use. In Section 3, we deal with problem (1)-(2). In Section 4, we deal with (3)-(4).

\section{Notions and Notations}

The fractional derivative can be defined in many ways (see, e.g., a classical book [12]) but they are all essentially equivalent. However, some definitions provide easier operations depending on a situation. Here, we shall use quite rudimentary way of defining the fractional derivative-via the notion of the Fourier multiplier operator. Let us first introduce the notion of the Fourier transform.

Definition 1. For a function $u \in L^{1}\left(\mathbb{R}^{d}\right) \cap L^{2}\left(\mathbb{R}^{d}\right)$ we define the Fourier transform $\mathscr{F}$ by

$$
\begin{array}{r}
\mathscr{F}(u)(\boldsymbol{\xi})=\widehat{u}(\boldsymbol{\xi})=\int_{\mathbb{R}^{d}} \exp (-2 \pi i \boldsymbol{\xi} \cdot \mathbf{x}) u(\mathbf{x}) d \mathbf{x}, \\
\boldsymbol{\xi} \cdot \mathbf{x}=\langle\boldsymbol{\xi}, \mathbf{x}\rangle=\sum_{j=1}^{d} \xi_{j} x_{j} .
\end{array}
$$

Using the Fourier transform, we can then define the Fourier multiplier operator through the following definition.
Definition 2. A (Fourier) multiplier operator $T_{\psi}: L^{2}\left(\mathbb{R}^{d}\right) \longrightarrow$ $L^{2}\left(\mathbb{R}^{d}\right)$ associated with a bounded function $\psi$ (see, e.g., [13]) is a mapping defined by

$$
T_{\psi}(u)=\mathscr{F}^{-1}(\psi \widehat{u}),
$$

where $\widehat{u}$ is the Fourier transform while $\mathscr{F}^{-1}$ (or ${ }^{\vee}$ ) is the inverse Fourier transform.

If, for a given $p \in(1, \infty)$, the multiplier operator $T_{\psi}$ satisfies

$$
\left\|T_{\psi}(u)\right\|_{L^{p}\left(\mathbb{R}^{d}\right)} \leq C\|u\|_{L^{p}\left(\mathbb{R}^{d}\right)}, \quad u \in L^{p}\left(\mathbb{R}^{d}\right),
$$

where $C$ is a positive constant, then its symbol $\psi$ is called an $L^{p}$ (Fourier) multiplier.

Finally, we can introduce the notion of the fractional derivative.

Definition 3. The fractional derivative $\partial_{x_{j}}^{\alpha}, j=1, \ldots, d, \alpha \in$ $\mathbb{R}$, is defined as the Fourier multiplier operator with symbol $\left(-2 \pi i \xi_{j}\right)^{\alpha}$ :

$$
\begin{array}{r}
\partial_{x_{j}}^{\alpha} u=T_{\left(-2 \pi i \xi_{j}\right)^{\alpha} u} \\
\mathscr{F}\left(\partial_{x_{j}}^{\alpha} u\right)=\left(-2 \pi i \xi_{j}\right)^{\alpha} \widehat{u} .
\end{array}
$$

The fractional Laplacean $\Delta^{\alpha}$ is defined as the Fourier multiplier operator with the symbol $-2 \pi|\xi|^{\alpha},|\xi|=\sqrt{\sum_{k=1}^{d} \xi_{k}^{2}}$ :

$$
\begin{aligned}
\Delta^{\alpha} u & =T_{-2 \pi|\xi|^{\alpha}} u \\
\mathscr{F}\left(\Delta^{\alpha} u\right) & =-2 \pi|\xi|^{\alpha} \widehat{u} .
\end{aligned}
$$

Such a definition is of course not optimal since $(-2 \pi i)^{\alpha}$ might not be unique. However, this does not affect the essence of our results and one can always fix one of the roots generated by $(-2 \pi i)^{\alpha}$.

Let us informally derive the integration by parts rule for the fractional derivatives. Formal derivation requires unsubstantial adaptations. We remark that we extend the involved functions by zero in domains where the functions are not defined. We have by means of the Plancherel theorem for smooth enough functions $u$ and $v$ vanishing at infinity (due to simplicity in notations, we present the rule in onedimensional situation):

$$
\begin{aligned}
\int v \partial^{\alpha} u & =\int \overline{\widehat{v}}(-2 \pi i \xi)^{\alpha} \widehat{u}=\int \overline{(-2 \pi i \xi)^{\alpha}} \overline{\widehat{v}} \widehat{u} \\
& =\int \overline{\mathscr{F}\left(T \overline{(-2 \pi i \xi)^{\alpha}}(v)\right.} \mathscr{F}(u)=\int T \overline{(-2 \pi i \xi)^{\alpha}}(v) u \\
& :=\int \overline{\partial^{\alpha}} v u
\end{aligned}
$$

where, as before, $T_{\overline{(-2 \pi i \xi)^{\alpha}}}=\overline{\partial^{\alpha}}$ is the Fourier multiplier operator with the symbol $\overline{(-2 \pi i \xi)^{\alpha}}$.

Now, we shall introduce the notion of controllability and observability and provide the theorems connecting the two notions. We will take the notations from $[4,9]$. 
Definition 4. We say that system (1) is controllable if for every $y_{0}, y_{1} \in \mathbb{R}^{d}$ there exists an $M$-component control vector function $u: \mathbb{R}^{+} \longrightarrow \mathbb{R}$ such that

$$
\int_{0}^{1} \partial_{t}^{\epsilon} y(T, \nu) d \nu=y_{1}
$$

for the prescribed final time $T>0$.

Interestingly, the latter definition is essentially equivalent to the following one taken from [9] and adapted to system (3) (see, e.g., [2, 3] for details).

Definition 5. Equation (3) is controllable in average with the cost $C$ if for all $y_{0}, y_{1}$ there exists $u$ such that

$$
\|u\|_{L^{2}\left((0, T) \times \mathbb{R}^{d}\right)} \leq C\left(\left\|y_{0}\right\|_{V}+\left\|y_{1}\right\|_{V}\right),
$$

where $H^{\alpha}\left(\mathbb{R}^{d}\right)$ is the Sobolev space of functions with the fractional derivative of order $\alpha$ and

$$
\int_{\mathbb{R}} y(T, v) d P(\nu)=y_{1} .
$$

Using a classical functional analytic tools such as HahnBanach theorem, one can also prove that controllability is equivalent to the observability. In order to introduce it, we need the notion of the adjoint problem to (3). Let $z \in L^{2}\left(\mathbb{R}^{d}\right)$ be a solution to

$$
\begin{aligned}
-\partial_{t} z & =A(\nu) \Delta^{\alpha} z \\
z(T, \mathbf{x}, \nu) & =z_{0} \in V^{\prime} .
\end{aligned}
$$

Indeed, using the Plancherel theorem, it is not difficult to check that

$$
\begin{aligned}
\int_{\mathbb{R}^{d}} \Delta^{\alpha} u z d \mathbf{x} & =\int_{\mathbb{R}^{d}} 2 \pi|\xi|^{\alpha} \widehat{u} \bar{z} d \xi=\int_{\mathbb{R}^{d}} u \overline{\Delta^{\alpha} z} d \mathbf{x} \\
& =\int_{\mathbb{R}^{d}} u \Delta^{\alpha} z d \mathbf{x}
\end{aligned}
$$

since $\Delta^{\alpha}$ is actually the multiplier operator with the even symbol and thus it is an operator mapping real functions into real functions (see [14, Lemma 5]). Thus, (14) is the corresponding adjoint equation to (3). We have the following definition of observability.

Definition 6. We say that system (3) is observable in average if there exists $C>0$ such that for all $z_{0} \in V^{\prime}$ it holds that

$$
\left\|z_{0}\right\|_{V^{\prime}} \leq C \int_{0}^{T}\left\|B^{*}(\nu) z(t, \mathbf{x}, \nu) d P(\nu)\right\|_{L^{2}\left(\mathbb{R}^{d}\right)} d t,
$$

where $z$ is the solution to (3) and $B^{*}$ is the adjoint operator to $B$.

In [9] one can find proof that the averaged controllability (Definition 5) is equivalent to the averaged observability (Definition 6) in a much more general situation where $A(\nu) \Delta^{\alpha}$ is replaced by a general operator $A(\nu)$ defined on a general Hilbert space $V$. Also, $u$ is taken to be from a Hilbert space $U$ while $B: U \longrightarrow V$ is a bounded functional from $U$ to $V$.

The following theorem holds.
Theorem 7 (see [9]). Problem (3) is controllable in average if and only if it is observable in average.

\section{Fractional ODE}

Although classical ODEs have been used for so many years as a tool for modeling of the systems with the applications in physics, biology, chemistry, medicine, and engineering, recent experiments indicate that there is a large class of complex systems with different kinetics which have a microscopic complex behavior, and their macroscopic dynamics cannot be described by classical models. We mention several contributions in different directions which is just a microsnapshot of the activities in this direction. Some examples can be found in the [15] where it has shown that fractional calculus models of viscoelastic materials are consistent with the physical principles that govern such materials. Concerning the signal processing and sampling theory in [16] authors derive an interpolation functions necessary for direct evaluation of fractional derivatives directly from the sample values. In mathematical modeling in biology several papers regarding the fractional-order differential models of biological systems with memory, such as dynamics of tumorimmune system and dynamics of HIV infection of CD4 $+\mathrm{T}$ cells and fractional-order predator-pray models, have been considered in [17-19]. Additional applications concern the spread of contaminants in underground water, network traffic, charge transport in amorphous semiconductors, cell diffusion process, the transmission of signals through strong magnetic fields such as those found within confined plasma, etc. A number of surveys with collections of applications can be found in [12].

In the case of system (1), the vector-valued function $y(t, v)=\left(y_{1}(t, v), \ldots, y_{d}(t, v)\right) \in \mathbb{R}^{d}$ is the state of the system, $A(\nu)$ is a $d \times d$-matrix governing its dynamics through the fractional ODE-system, and $u(t)$ is a $N$-component control vector in $\mathbb{R}^{N}$ acting on the system through the control matrix $B(\nu) \in M^{d \times N}$ depending on the parameter $\nu$.

The matrices $A$ and $B$ are measurable and bounded with respect to $v \in(0,1)$. We remark that the initial state $y_{0}$ as well as the final state $y_{1}$ are required to be independent of $v$, while it is obviously not a case with the solution $y$ of (1).

As usual in the control-type problems, we shall need the adjoint system in order to use the observability concept. The adjoint system in the case of system (1) has the form

$$
\begin{aligned}
-\partial_{t} \overline{\partial_{t}^{\epsilon}} z & =A^{*}(\nu) z \\
\left.z\right|_{t=T} & =z_{0},
\end{aligned}
$$

where $A^{*}(\nu)$ is the adjoint matrix to the matrix $A$.

Indeed, we have for an arbitrary function $z \in H^{\alpha}([0, T])$ using (10) (we remind the reader again that we extend the functions by zero on the sets where they are not defined, i.e., out of the interval $[0, T])$ :

$$
\int_{0}^{T} \partial_{t}^{\alpha} y z d t=\int_{0}^{T} A(\nu) y z d t \Longrightarrow
$$




$$
\begin{aligned}
& \int_{0}^{T} \partial_{t}^{1+\epsilon} y z d t=\int_{0}^{T} y A^{*}(\nu) z d t \Longrightarrow \\
& \int_{0}^{T} \partial_{t}\left(\partial_{t}^{\epsilon} y\right) z d t=\int_{0}^{T} y A^{*}(\nu) z d t \Longrightarrow \\
& \left.\partial_{t}^{\epsilon} y z\right|_{0} ^{T}-\int_{0}^{T} \partial_{t}^{\epsilon} y \partial_{t} z d t=\int_{0}^{T} y A^{*}(\nu) z d t \Longrightarrow \\
& \left.\partial_{t}^{\epsilon} y z\right|_{0} ^{T}-\int_{0}^{T} \partial_{t}^{\epsilon} y z^{\prime} d t=\int_{0}^{T} \partial_{t}^{\epsilon} y \overline{\partial_{t}^{-\epsilon}}\left(A^{*}(\nu) z\right) d t \Longrightarrow \\
& -\partial_{t} z=\overline{\partial_{t}^{-\epsilon}}\left(A^{*}(\nu) z\right), \\
& \left.z\right|_{t=T}=z_{0} .
\end{aligned}
$$

Applying the operator $\overline{\partial_{t}^{\epsilon}}$ here, we reach (17). Following [9, Definition 2.3.5] and [4, p.4 (10)], we have the following averaged observability inequality for the adjoint system (17):

$$
\left|z_{0}\right|^{2} \leq C \int_{0}^{T}\left|\int_{0}^{1} B^{*}(\nu) z(t, v) d \nu\right|^{2} d t
$$

for all $z_{0} \in \mathbb{R}^{d}$. We have the following theorem.

Theorem 8. System (1) fulfills the averaged controllability property from Definition 5 if and only if the adjoint system (17) satisfies (19) and both are equivalent to the rank conditions

$$
\operatorname{rank}\left[\int_{0}^{1}[A(\nu)]^{j} B(\nu) d \nu: j \geq 0\right]=d .
$$

When these properties hold, the averaged control of minimal $L^{2}\left([0, T] ; R^{d}\right)$-norm is given by

$$
u(t)=\int_{0}^{1} B^{*}(\nu) z(t, v) d v
$$

where $z$ is the solution of the adjoint system (17) corresponding to the datum $z_{0}$ minimizing the functional

$$
\begin{aligned}
J\left(z_{0}\right)= & \frac{1}{2} \int_{0}^{T}\left|\int_{0}^{1} B^{*}(\nu) z(t, v) d \nu\right|^{2} d t-\left\langle y_{1}, z_{0}\right\rangle \\
& +\left\langle y_{0}, \int_{0}^{1} z(0, v) d \nu\right\rangle .
\end{aligned}
$$

Proof. We have for a solution $z$ to (17) (see the derivation (18) above)

$$
\begin{aligned}
& \int_{0}^{T} \int_{0}^{1}\langle B(v) u(t), z(t, v)\rangle d v d t \\
& \quad=\int_{0}^{T} \int_{0}^{1}\left\langle\partial_{t}^{\alpha} y-A(v) y, z\right\rangle d v d t= \\
& \int_{0}^{1}\left\langle\partial_{t}^{\epsilon} y(T, v) d v, z_{0}\right\rangle-\int_{0}^{1}\left\langle y_{0}, z(0, v)\right\rangle d v \\
& \quad+\int_{0}^{1} \int_{0}^{T}\left\langle\partial_{t}^{\epsilon} y,-\partial_{t} z+A^{*}(v) z\right\rangle d t d v=\left\langle y_{1}, z_{0}\right\rangle \\
& \quad-\int_{0}^{1}\left\langle y_{0}, z(0, v)\right\rangle d v
\end{aligned}
$$

according to the controllability condition from Definition 5. In other words, Definition 5 is recasted as

$$
\begin{aligned}
\left\langle y_{1}, z_{0}\right\rangle= & \int_{0}^{1}\left\langle y_{0}, z(0, v)\right\rangle d v \\
& +\int_{0}^{T} \int_{0}^{1}\langle B(v) u(t), z(t, v)\rangle d v d t
\end{aligned}
$$

which is the Euler-Lagrange equation associated with the minimization of the functional $J$ from (22) (see $[4$, p.4. (14)]. Now, the proof follows the steps from the proof of $[4$, Theorem 3].

3.1. Numerical Example. At this time, we want to give a simple numerical illustration of the theory that we have presented so far. The most difficult issue here is to determine the final value (at $t=T$ ) for the adjoint problem (17) since it is equal to the datum minimizing the functional (22). This means that we essentially need to know explicit dependence of $z(t, v)$ and $z(0, v)$ from $z_{0}$ and then we can apply the Euler-Lagrange equations techniques. Here, we shall simply assume that we have a minimizing final value $z_{0}$ for the adjoint state, compute the control function $u$ using (21), and then see what final value we can get. In the further research, we shall develop a numerical method in the frame of which we are able to solve the original control problem (finding control $u$ from the equations, initial and final states).

Here, we aim to solve

$$
\begin{aligned}
& \partial_{t}^{\alpha} y(t, \nu)=A(\nu) y(t, \nu)+B(\nu) u(t), \quad 1<\alpha<2, \\
& \partial_{t}^{\epsilon} y(0, \nu)=y^{0} \in \mathbb{R}^{d},
\end{aligned}
$$

where $A$ and $B$ are as as follows:

$$
\begin{aligned}
& A(\nu)=\left[\begin{array}{cc}
v & 1-v \\
1-v & v
\end{array}\right], \\
& B(\nu)=\left[\begin{array}{ll}
\nu & 0 \\
0 & v
\end{array}\right] .
\end{aligned}
$$

As for the adjoint system (17), we shall assume that $z_{0}=(1,9)$. We then devise the following strategy.

Let matrices $A$ and $B$ corresponding to system (25) of order $\alpha$ and initial condition be given.

(1) Formulate the adjoint problem (17).

(2) Solve (as will be described later) the adjoint problem and obtain the solution $z$.

(3) Use formula (21) to compute the averaged control $u$.

So the only challenge now is to solve step (2), that is, to solve fractional-order ODE. To this end we will use GrunwaldLetnikov discretization; for further reference on modern numerical treatment of the fractional-order ODE please see [20]. 
Definition 9. Let $\alpha>0$. The operator

$$
D_{G L}^{\alpha} u(x)=\lim _{\Delta x \longrightarrow 0} \frac{1}{\Delta x^{\alpha}} \sum_{k=0}^{m} w_{k} u(x-k \Delta x),
$$

$$
a \leq x \leq b,
$$

where $m=(x-a) / \Delta x$ and $\omega_{k}=(-1)^{k}\left(\begin{array}{c}\alpha \\ k\end{array}\right)$, is called the Grunwald-Letnikov fractional derivative of order $\alpha$.

This definition holds for arbitrary functions $u$, but the convergence of the infinite sum cannot be ensured for all functions [21]. After employing Grunwald-Letnikov discretization in the case of $2 \times 2$ system $z=\left[z^{1}, z^{2}\right]^{\tau}$ we have

$$
\begin{aligned}
& \frac{1}{\Delta x^{\alpha}} w_{0} z_{n}^{1}=A_{1,1}^{*} z_{n}^{(1)}+A_{1,2}^{*} z_{n}^{(2)}-\frac{1}{\Delta x^{\alpha}} \sum_{k=1}^{m} w_{k} z_{n-k}^{1} \\
& \frac{1}{\Delta x^{\alpha}} w_{0} z_{n}^{2}=A_{2,1}^{*} z_{n}^{(1)}+A_{2,2}^{*} z_{n}^{(2)}-\frac{1}{\Delta x^{\alpha}} \sum_{k=1}^{m} w_{k} z_{n-k}^{2}
\end{aligned}
$$

After solving with respect to $z_{n}^{1}$ and $z_{n}^{2}$ we obtain the finite difference scheme for computing step (2).

$$
\begin{aligned}
& z_{n}^{(1)} \\
& =-\frac{1}{c_{1} c_{3}}\left(\frac{1}{c_{2}} A_{1,2}^{*} \frac{1}{\Delta x^{\alpha}} \sum_{k=1}^{m} w_{k} z_{n-k}^{(2)}+\frac{1}{\Delta x^{\alpha}} \sum_{k=1}^{m} w_{k} z_{n-k}^{(1)}\right) \\
& z_{n}^{(2)} \\
& =-\frac{1}{c_{2} c_{3}}\left(\frac{1}{c_{1}} A_{2,1}^{*} \frac{1}{\Delta x^{\alpha}} \sum_{k=1}^{m} w_{k} z_{n-k}^{(1)}+\frac{1}{\Delta x^{\alpha}} \sum_{k=1}^{m} w_{k} z_{n-k}^{(2)}\right)
\end{aligned}
$$

where the coefficients are given by the following formulas:

$$
\begin{aligned}
& c_{1}=\frac{1}{\Delta x^{\alpha}} \omega_{0}-A_{1,1}^{*}, \\
& c_{2}=\frac{1}{\Delta x^{\alpha}} \omega_{0}-A_{2,2}^{*}, \\
& c_{3}=1-\frac{1}{c_{1} c_{2}} A_{1,2}^{*} A_{2,1}^{*} .
\end{aligned}
$$

By assuming that $y_{0}=(0,1)$ we see that we reach the final state $y_{1}=(0.5,23)$. The result is plotted on Figure 1 .

\section{Fractional Diffusion Equation}

A fundamental result in Einstein's theory of Brownian motion is that the mean square displacement of the Brownian particle scales linearly with time and the probability density function for Brownian motion is the normal distribution. This is not the case in the numerous situations where standard diffusion does not capture the physical process, i.e., when the mean square displacement scales as a fractional power law in time. Such type of diffusion has been suggested as a concise model for the description of the dynamic events that occur in biological tissues and in [22] authors consider cancer tumor models and investigate the need for fractionalorder derivative as compared to the classical first order derivative in time. In [23] authors have shown, using of fluorescence correlation spectroscopy, that the mean square displacement of a protein in folding will grow less than linear in time and the degree of this anomaly depends on the size and conformation of the traced particle and on the total protein concentration of the solution. Several more examples of the fractional-order equations encompass processes that describe anomalous diffusion in electrolytic cell [24] and on fractals (physical objects of fractional dimension, like some amorphous semiconductors or strongly porous materials; see $[25,26]$ and references therein), fractional random walk, etc.

Control problem for fractional diffusion type equation has already been considered in [27] in the case when the fractional operator acts on the time variable. Here, we are going to consider the fractional diffusion equation with the fractional Laplacean. This type of equation has been widely considered (see [11] and references therein) and it is usually interpreted as the interpolation between hyperbolic and parabolic equations. Here, we shall prove a controllability result (Definition 5) analogical with the one from [9].

To this end, we shall use Theorem 7 stating that the controllability (Definition 5) and observability (Definition 6) are equivalent. Remark that the observability inequality involves the solution $z$ to the adjoint problem (14) in whose formulation the operator $B$ does not take part. However, the observability inequality actually connects the function $z$ and the operator $B$ which implies necessity to impose a relation between function $A$ appearing in (3) and operator $B$. Indeed, if, for instance, $B \equiv 0$ then we will not be able to control (3) through any function $u$. Remark that in the case of (1) this type of condition was the rank condition (20).

Before we introduce an adaptation of the rank condition to our situation, we shall determine space $V$ in which we shall choose our initial data. We denote for the fixed $T>0$ and $M>\|A\|_{L^{\infty}(\Omega)}$

$$
V=\left\{f \in L^{2}\left(\mathbb{R}^{d}\right):\left\|\widehat{f} e^{M T|\xi|^{\alpha}}\right\|_{L^{2}\left(\mathbb{R}^{d}\right)}<\infty\right\}
$$

Clearly, dual space $V^{\prime}$ is of the form

$$
V^{\prime}=\left\{f \in L^{2}\left(\mathbb{R}^{d}\right):\left\|\widehat{f} e^{-M T|\xi|^{\alpha}}\right\|_{L^{2}\left(\mathbb{R}^{d}\right)}<\infty\right\}
$$

Scalar product on the space $V$ is given by

$$
\langle u, v\rangle=\int_{\mathbb{R}^{d}} \widehat{u}(\boldsymbol{\xi}) \widehat{v}(\xi) \exp \left(2 M T|\xi|^{\alpha}\right) d \xi
$$

and on $V^{\prime}$ by

$$
\langle u, v\rangle=\int_{\mathbb{R}^{d}} \widehat{u}(\xi) \widehat{v}(\xi) \exp \left(-2 M T|\xi|^{\alpha}\right) d \xi .
$$

We introduce the following relation between $A$ and $B$ which we call the rank condition by the analogy with (20). 

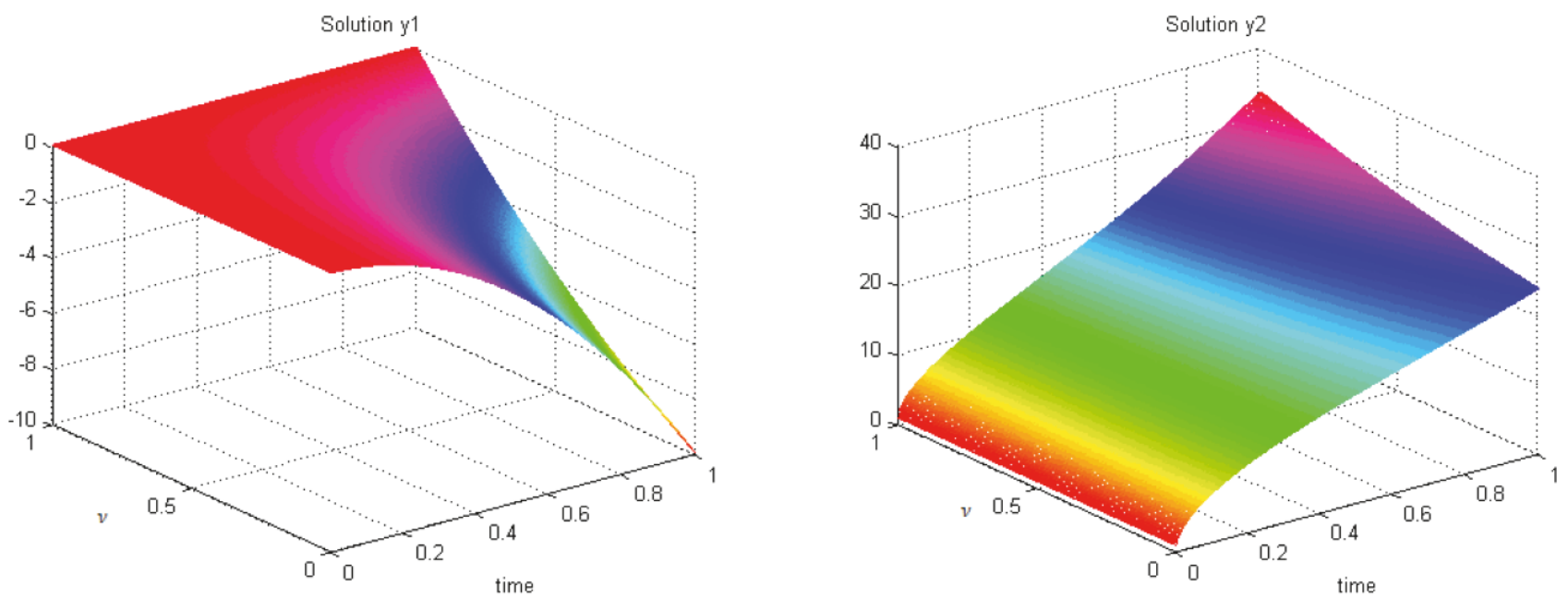

(a) Solution of the example $y=\left[y_{1}, y_{2}\right]^{\tau}$
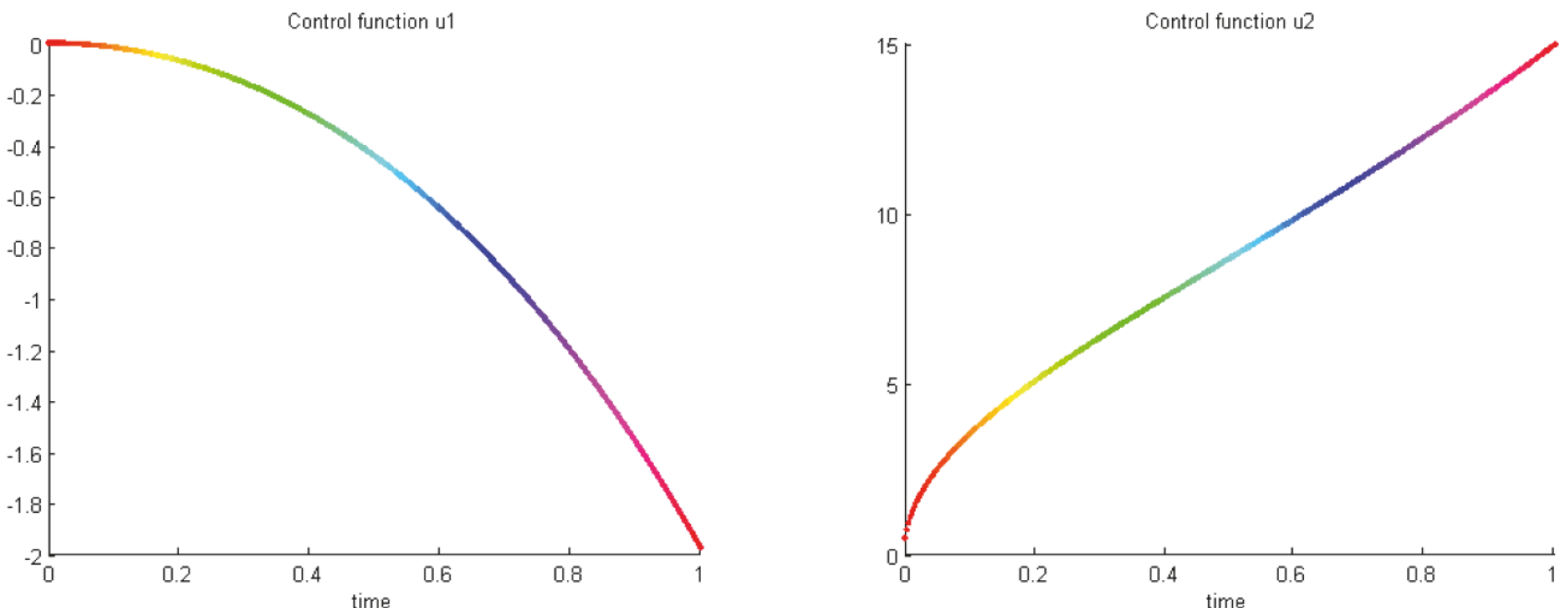

(b) Computed control function $u=[u 1, u 2]^{\tau}$

FIGURE 1: The control function $u=\left(u_{1}, u_{2}\right)$ and the solution $y=\left(y_{1}, y_{2}\right)$ to $(25)$ under the assumption that $z_{0}=(1,9)$ minimizes $(22)$.

Definition 10. We say that function $A$ and operator $B$ satisfy the rank condition if there exists a constant $C>0$ such that for every $z \in L^{2}\left((0, T] \times \mathbb{R}^{d} ; L^{1}(\Omega ; d P)\right)$ it holds that

$$
\begin{aligned}
& \left\|\int_{\Omega} B^{*}(\nu) z(t, \cdot, \nu) d P(\nu)\right\|_{L^{2}\left(\mathbb{R}^{d}\right)} \\
& \quad \geq \frac{1}{C}\left\|\int_{\Omega} z(t, \cdot, v) d P(\nu)\right\|_{L^{2}\left(\mathbb{R}^{d}\right)} \quad \text { for every } t \geq 0 .
\end{aligned}
$$

Using this condition, we can prove the observability of problem (3)-(4).

Theorem 11. Under conditions (37), problem (3)-(4) is observable.
Proof. Let us first find the solution to (14). We have after applying the Fourier transform on (14)

$$
\begin{aligned}
\partial_{t} \widehat{z} & =A(\nu)|\xi|^{\alpha} \widehat{z} \Longrightarrow \\
\widehat{z} & =\widehat{z_{0}} \exp \left(-A(\nu)|\xi|^{\alpha}(T-t)\right) ;
\end{aligned}
$$

i.e.,

$$
\widehat{z_{0}}(t, \xi)=\widehat{z}(t, \xi, \nu) \exp \left(A(\nu)|\xi|^{\alpha}(T-t)\right) .
$$

Assume without losing generality that $\widehat{u}$ is purely real function. Otherwise, we simply split consideration below on real part of $\widehat{u}$ and imaginary part of $\widehat{u}$.

Next, under the assumption that $\widehat{u}$ is real, we split considerations on two domains:

$$
\begin{aligned}
& \Omega_{t, \xi}^{+}=\{v: \widehat{z}(t, \boldsymbol{\xi}, v) \geq 0\}, \\
& \Omega_{t, \xi}^{-}=\{v: \widehat{z}(t, \boldsymbol{\xi}, v) \leq 0\} .
\end{aligned}
$$


We fix $(t, \xi)$ and consider (39) first on $\Omega_{t, \xi}^{+}$. It holds that

$$
\begin{aligned}
& \widehat{z_{0}}(t, \xi) \exp \left(M T|\xi|^{\alpha}\right) \text { meas }\left(\Omega_{t, \xi}^{+}\right) \\
& \quad \geq\left|\int_{\Omega_{t, \xi}^{+}} \widehat{z_{0}}(t, \xi)\right| d P(\nu) \\
& \quad=\left|\int \widehat{z}(t, \xi, \nu) \exp \left(A(\nu)|\xi|^{\alpha}(T-t)\right) d P(\nu)\right| \\
& \quad \geq\left|\int_{\Omega_{t, \xi}^{+}} \widehat{z}(t, \xi, \nu) d P(\nu)\right| .
\end{aligned}
$$

In the domain $\Omega_{t, \xi}^{-}$, we have

$$
\begin{aligned}
& \left|\widehat{z_{0}}(t, \xi) \exp \left(M T|\xi|^{\alpha}\right)\right| \operatorname{meas}\left(\Omega_{t, \xi}^{-}\right) \\
& \quad \geq\left|\int_{\Omega_{t, \xi}^{-}} \widehat{z_{0}}(t, \xi) \exp \left(A(\nu) T|\xi|^{\alpha}\right) d P(\nu)\right| \\
& \quad=\left|\int_{\Omega_{t, \xi}^{-}} \widehat{z}(t, \xi, \nu) \exp \left(-A(\nu)|\xi|^{\alpha} t\right) d P(\nu)\right| \\
& \quad \geq\left|\int_{\Omega_{t, \xi}^{-}} \widehat{z}(t, \xi, \nu) d P(\nu)\right| .
\end{aligned}
$$

Summing (41) and (42), we get

$$
\left|\widehat{z_{0}}(t, \xi) \exp \left(M T|\xi|^{\alpha}\right)\right| \geq\left|\int_{\Omega} \widehat{z}(t, \xi, \nu) d P(\nu)\right| .
$$

Finding the $L^{2}\left(\mathbb{R}^{d}\right)$-norms of the both sides of the later inequality with respect to $\xi \in \mathbb{R}^{d}$, and applying the Plancherel theorem, we get

$$
\left\|z_{0}(t, \cdot)\right\|_{V^{\prime}} \geq\left\|\int_{\Omega} z(t, \cdot, \nu) d P(\nu)\right\|_{L^{2}\left(\mathbb{R}^{d}\right)} .
$$

Finally, integrating the latter over $[0, T]$ and applying (37), we prove the observability inequality.

From the previous theorem and Theorem 7, one has the following.

Theorem 12. Problem (3)-(4) is controllable in average in the sense of Definition 5.

As for the numerical strategy for reaching the control $u$ for (3)-(4), we remark that we can essentially use the strategy used for the ODE-problem. Namely, since the solution $y$ to (3) belongs to the $L^{2}\left(\mathbb{R}^{d}\right)$-valued space, we can rewrite it in the form

$$
y(t, \mathbf{x}, v)=\sum_{j=1}^{\infty} u_{j}(t, v) e_{j}(\mathbf{x})
$$

where $\left\{e_{j}\right\}_{j \in \mathbb{N}}$ form an orthonormal basis in $L^{2}\left(\mathbb{R}^{d}\right)$. Moreover, $\left\{e_{j}\right\}_{j \in \mathbb{N}}$ can be chosen to be eigenvalues of the fractional
Laplace operator $\Delta^{\alpha}$. Thus, (3) can be rewritten as an infinite system of ODEs:

$$
\partial_{t} u_{j}=A(\nu) \lambda_{j} u_{j}+\sum_{i=1}^{\infty} b_{i j}(\nu) u_{i}(t, v),
$$

where $B(\nu) e_{j}=\sum_{i=1}^{\infty} b_{i j}(\nu) e_{i}$. Then, we simply solve the problem for the finite dimensional approximation of the system to get an approximation of the control $u$.

\section{Data Availability}

No data were used to support this study.

\section{Conflicts of Interest}

The authors declare that they have no conflicts of interest.

\section{Acknowledgments}

The research is supported in part by the COST action 15225: Fractional-order systems-analysis, synthesis and their importance for future design. Part of the research was prosecuted during the visit of Darko Mitrovic to the University of Sarajevo whose hospitality we gratefully acknowledge. The research is also supported by the Croatian Science Foundation under Project 9780 WeConMApp.

\section{References}

[1] J.-L. Lions, Controlabilité Exacte, Stabilisation et Perturbations de Systemes Distribués, vol. 8, Controlabilité Exacte, Masson, Paris, 1988.

[2] M. Tucsnak and G. Weiss, Observation and Control for Operator Semigroups, Advanced Texts, Birkhäuser, Switzerland, 2009.

[3] E. Zuazua, "Controllability and observability of partial differential equations: some results and open problems," in Handbook of Differential Equations: Evolutionary Equations, vol. 3 of Handb. Differ. Equ., pp. 527-621, Elsevier, North-Holland, Amsterdam, 2007.

[4] E. Zuazua, "Averaged control," Automatica, vol. 50, no. 12, pp. 3077-3087, 2014.

[5] J. Lohéac and E. Zuazua, "From averaged to simultaneous controllability of parameter dependent finite-dimensional systems," Annales de la Faculté des Sciences de Toulouse Mathématiques, vol. 25, no. 6, pp. 785-828, 2016.

[6] Q. Lu and E. Zuazua, "Averaged controllability for random evolution partial differential equations," Journal de Mathematiques Pures et Appliquées, vol. 105, no. 3, pp. 367-414, 2016.

[7] M. Lazar and E. Zuazua, "Averaged control and observation of parameter-depending wave equations," Comptes Rendus Mathématique, vol. 352, pp. 497-502, 2014.

[8] M. Lazar, "Stability of observations of partial differential equations under uncertain perturbations," ESAIM: Control, Optimisation and Calculus of Variations, 2018.

[9] J. Coulson, Average Controllability of Random Heat Equations [Ph.D. thesis], Queen's University Kingston, Ontario, Canada, 2017. 
[10] K. Li, J. Peng, and J. Jia, "Cauchy problems for fractional differential equations with Riemann-Liouville fractional derivatives," Journal of Functional Analysis, vol. 263, no. 2, pp. 476-510, 2012.

[11] L. Vázquez, J. J. Trujillo, and M. P. Velasco, "Fractional heat equation and the second law of thermodynamics," Fractional Calculus and Applied Analysis, vol. 14, no. 3, pp. 334-342, 2011.

[12] I. Podlubny, Fractional Differential Equations: An Introduction to Fractional Derivatives, Academic Press, New York, NY, USA, 1999.

[13] L. Grafakos, Classical Fourier analysis, vol. 249 of Graduate Texts in Mathematics, Springer Science and Business Media, 2008.

[14] M. Misur and D. Mitrovic, "On a generalization of compensated compactness in the $L^{p} L^{q}$ setting," Journal of Functional Analysis, vol. 268, no. 7, pp. 1904-1927, 2015.

[15] R. L. Bagley and P. J. Torvik, "A theoretical basis for the application of fractional calilus to visoelasticity," Journal of Rheology, vol. 27, no. 3, pp. 201-210, 1983.

[16] R. J. Marks and M. W. Hall, "Differintegral interpolation from a bandlimited signal's samples," IEEE Transactions on Acoustics, Speech, and Signal Processing, vol. 29, no. 4, pp. 872-877, 1981.

[17] E. Ahmed, A. M. A. El-Sayed, and H. A. A. El-Saka, "Equilibrium points, stability and numerical solutions of fractionalorder predator-prey and rabies models," Journal of Mathematical Analysis and Applications, vol. 325, no. 1, pp. 542-553, 2007.

[18] F. A. Rihan, "Numerical modeling of fractional-order biological systems," Abstract and Applied Analysis, vol. 2013, Article ID 816803, 11 pages, 2013.

[19] H.-L. Li, L. Zhang, C. Hu, Y.-L. Jiang, and Z. Teng, "Dynamical analysis of a fractional-order predator-prey model incorporating a prey refuge," Applied Mathematics and Computation, vol. 54, no. 1-2, pp. 435-449, 2017.

[20] N. J. Ford and J. A. Connolly, "Comparison of numerical methods for fractional differential equations," Communications on Pure and Applied Analysis, vol. 5, no. 2, pp. 289-306, 2006.

[21] M. Weilbeer, Efficient numerical methods for fractional differential equations and their analytical background [Ph.D. thesis], Technischen Universitat Braunschweig, 2005.

[22] O. S. Iyiola and F. D. Zaman, "A fractional diffusion equation model for cancer tumor," AIP Advances, vol. 4, no. 10, Article ID 107121, 2014.

[23] M. Weiss, M. Elsner, F. Kartberg, and T. Nilsson, "Anomalous subdiffusion is a measure for cytoplasmic crowding in living cells," Biophysical Journal, vol. 87, no. 5, pp. 3518-3524, 2004.

[24] E. K. Lenzi, L. R. Evangelista, and G. Barbero, "Fractional diffusion equation and impedance spectroscopy of electrolytic cells," The Journal of Physical Chemistry B, vol. 113, no. 33, pp. 1137111374, 2009.

[25] V. V. Anh and N. N. Leonenko, "Spectral analysis of fractional kinetic equations with random data," Journal of Statistical Physics, vol. 104, no. 5-6, pp. 1349-1387, 2001.

[26] R. Metzler and J. Klafter, "The random walk's guide to anomalous diffusion: a fractional dynamics approach," Physics Reports, vol. 339, no. 1, pp. 1-77, 2000.

[27] G. M. Mophou, "Optimal control of fractional diffusion equation," Computers \& Mathematics with Applications, vol. 61, no. 1, pp. 68-78, 2011. 


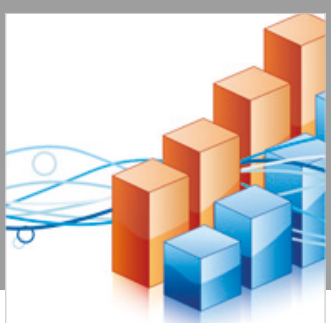

Advances in

Operations Research

\section{-n-m}
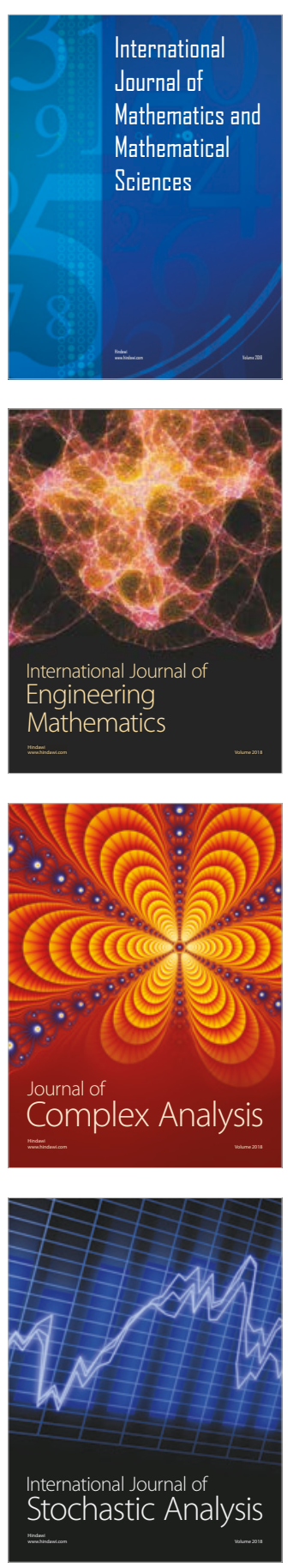
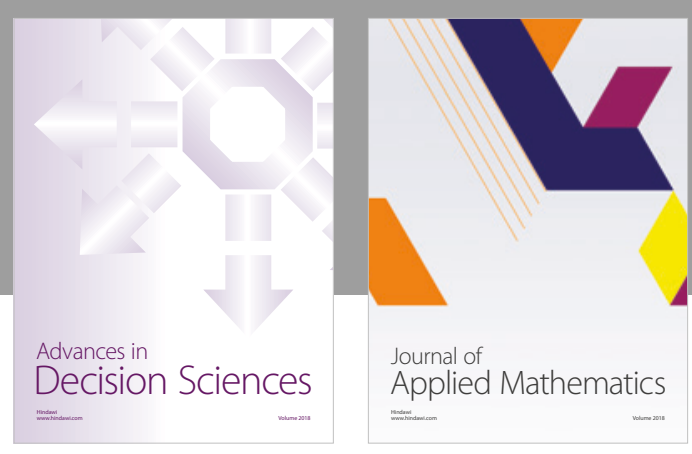

Journal of

Applied Mathematics
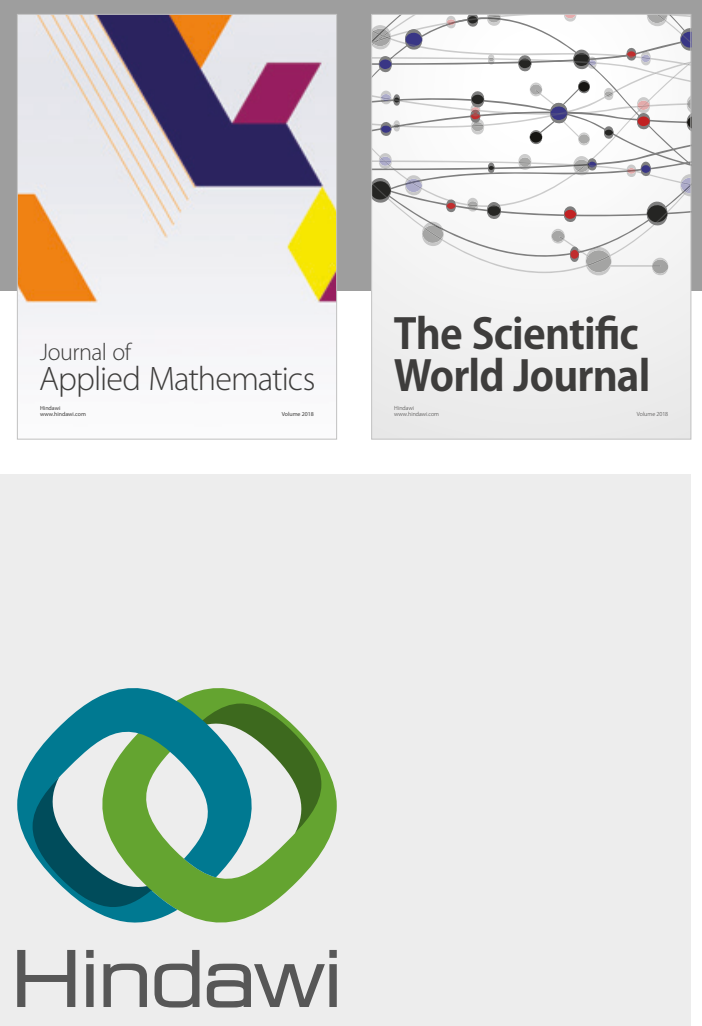

Submit your manuscripts at

www.hindawi.com

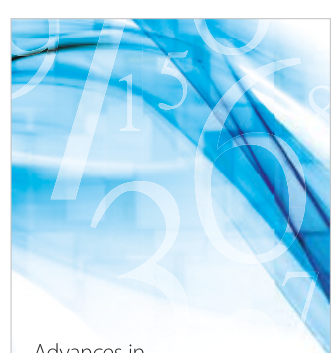

Advances in
Numerical Analysis
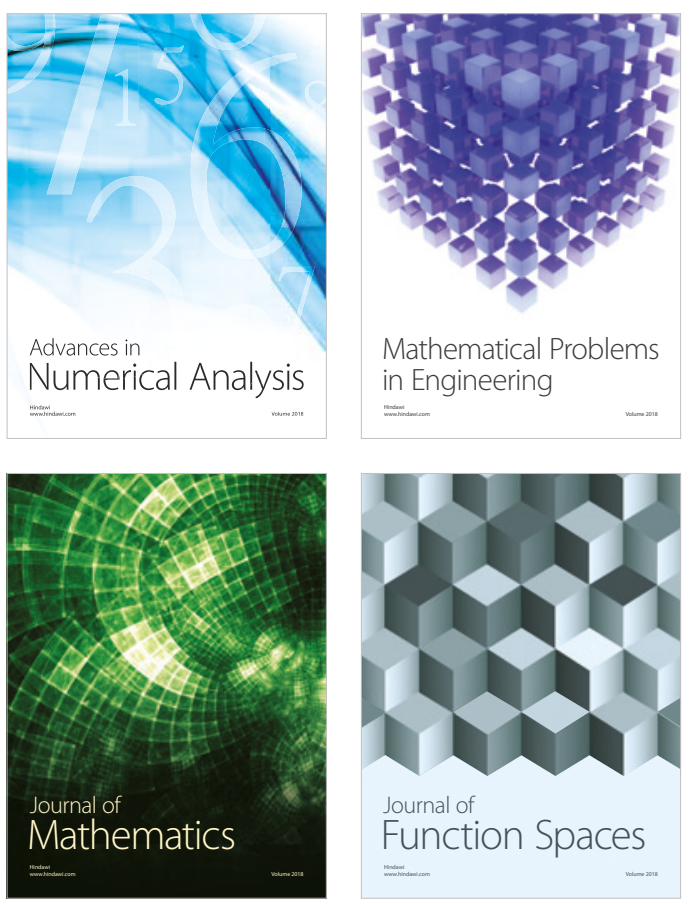

Mathematical Problems in Engineering

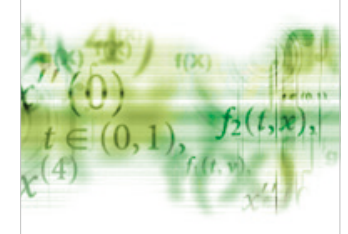

International Journal of

Differential Equations

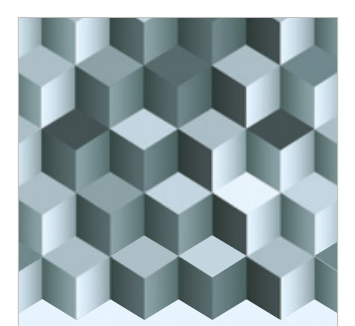

Journal of

Function Spaces

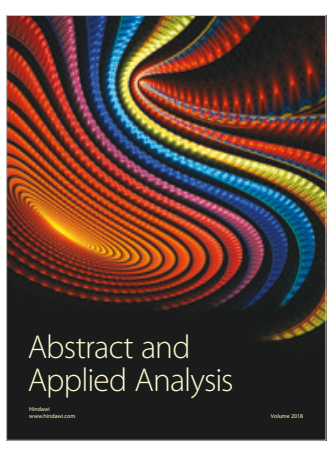

The Scientific

World Journal

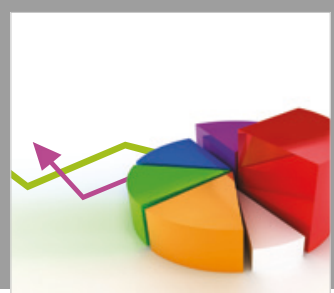

Journal of

Probability and Statistics
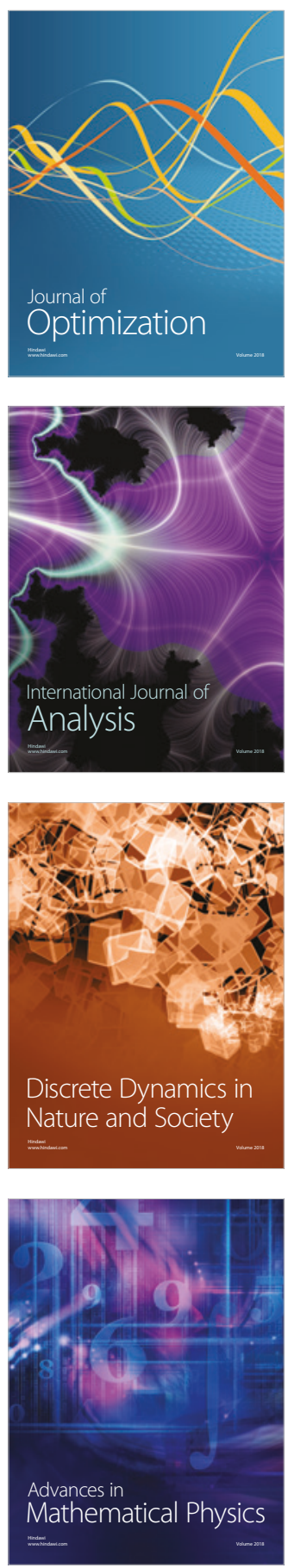